\title{
Flexible Parametric Models for Relative Survival, with Application in Coronary Heart Disease
}

\author{
Christopher P Nelson \\ Centre for Biostatistics and Genetic Epidemiology, Department of Health Sciences, \\ University of Leicester, UK. \\ Email: cn46@le.ac.uk \\ Paul C Lambert \\ Centre for Biostatistics and Genetic Epidemiology, Department of Health Sciences, \\ University of Leicester, UK. \\ Iain B Squire \\ Department of Cardiovascular Sciences, University of Leicester, UK \\ David R Jones \\ Centre for Biostatistics and Genetic Epidemiology, Department of Health Sciences, \\ University of Leicester, UK.
}




\section{SUMMARY}

Relative survival is frequently used in population based studies as a method for estimating disease-related mortality without the need for information on cause of death. We propose an extension to relative survival of a flexible parametric model proposed by Royston and Parmar for censored survival data. The model provides smooth estimates of the relative survival and excess mortality rates by using restricted cubic splines on the log cumulative excess hazard scale. The approach has several advantages over some of the more standard relative survival models, which adopt a piecewise approach, the main being the ability to model time on a continuous scale, the survival and hazard function are obtained analytically and it does not use split-time data. 


\section{INTRODUCTION}

Survival data are often modelled using a Cox proportional hazards model, which has the advantage of estimating covariate effects as log hazard ratios without the need to estimate the baseline hazard. However, the behaviour of the hazard function may be of medical interest because it is directly related to the time-course of an illness. The baseline hazard rate can also help in understanding the natural history of the disease through the way the hazard rate changes over time. Selected parametric models such as the Weibull model are an alternative to the Cox model. However, these models are not as popular in practice due to concerns over the restrictions imposed on the shape of the hazard function. A flexible parametric model using restricted cubic splines was proposed by Royston and Parmar[1] for censored survival data, enabling the baseline hazard to be directly modelled. The restricted cubic splines offer greater flexibility in the shape of the hazard function when compared to standard parametric models. In addition they can be easily extended to time dependent effects. One of the key advantages of the method is that the survival and hazard functions are obtained analytically speeding up computational time over methods adopting numerical integration. The application of the method has so far been limited[24] although it is available to run using Stata[5]. Here we extend the flexible parametric models to relative survival models.

Relative survival $R(t)$ is defined as the ratio of the observed, all-cause, survival $S(t)$ with the expected survival $S^{*}(t)[6]$ :

$$
R(t)=S(t) / S^{*}(t)
$$


So that on the hazard scale the overall hazard rate, $h(t)$ is given by

$$
h(t)=h^{*}(t)+\lambda(t)
$$

where $h^{*}(t)$ is the expected mortality/hazard rate and $\lambda(t)$ is the excess mortality rate associated with the disease of interest. The expected mortality rate is assumed known, often obtained from routinely collected data and matched to the population under study by age, sex, time period and potentially other factors. Relative survival is usually estimated using life tables, but there has been recent interest in models for relative survival.

Comparison with an expected mortality in the population is the key difference between relative survival and the standard methods in that it provides a measure of mortality associated with a disease without the need for information on cause of death. Causespecific analysis can be an alternative; however the cause of death is often difficult to state with certainty leading to misclassification. In population based research, for example, determining the cause of death is usually obtained from death certificates which can be unreliable in both cancer and heart disease[7, 8].

Relative survival is used routinely in population based cancer studies[9]. Use of relative survival in therapeutic areas other than cancer is limited though there are a few applications of the idea for survival following heart surgery [10, 11], after a myocardial infarction (MI) [12, 13] and after stroke[14, 15]. 
There have been several approaches proposed to model $\lambda(t)$. The most commonly used in applied research is a piecewise approach wherein there is a need to split the timescale. Hakulinen and Tenkanen[16] estimate the model based on grouped data using a generalised linear model (GLM) framework with a binomial assumption for the number of observed deaths. Estève et al.[17] adopted a full likelihood approach based on individual level data. Dickman et al.[18] proposed a GLM with a Poisson error structure using exact survival times and individual level data which is equivalent to the Estève et al. full likelihood approach. Models can also be fitted at the grouped level (i.e. collapsed over categorical covariate patterns). Relative survival models have been extended to more flexible models such as multivariate fractional polynomials (MFP)[19] and cubic splines[20] that also use split-time data. B-splines have also been suggested for relative survival to model the hazard ratio as a flexible function of time by Giorgi et al.[21] as have restricted cubic splines by Bolard et al.[22], both using non-split time data.

The individual level piecewise approach yields several observations for each individual in the dataset as there is a record for each subdivision of time. This can have computational implications in very large datasets. For example Lambert et al.[19] used grouped data when fitting fractional polynomial models due to the computation time needed for individual level models. A disadvantage of using grouped data is that continuous covariates need to be categorised, which is not a preferred practice due to the loss of power[23] and subjectivity in choice of cut-points. Piecewise models rely on a subjective choice of the number and location of split points and lack clinical plausibility. Using a piecewise approach also implies generation of many parameters for time dependent effects (if a dichotomous covariate is perceived to vary over time then there is 
a need to create (h-1) more parameters where $h$ is the number of splits in the timescale. It is possible to simplify this by making assumptions, for example that excess hazards are proportional after 2 years. B-splines[21], like the restricted cubic spline approach proposed by Bolard et al.[22], obtain the survival function using numerical integration increasing computation time. All current approaches smooth on the log excess hazard scale.

The flexible parametric approach we adopt models on the log cumulative excess hazard scale, which can have advantages in terms of model stability. The hazard and survival function are obtained analytically and non-split time data is used, i.e. a single record per subject. The models are thus restricted to only one time scale. This paper proposes an extension of the Royston and Parmar flexible parametric model[1] to relative survival. This method is illustrated in a heart disease dataset for the Leicestershire area in the UK in which modelling the difference between three age groups will be illustrated. In section 2 the methodology of relative survival and the extension of Royston and Parmar's method are described. The dataset is described in section 3. Section 4 details analyses of the dataset focusing on the fit of the spline models and a comparison with standard relative survival analyses. Section 5 is a discussion of implications along with the advantages and limitations of this method. 


\section{METHODS}

In survival analysis an individual's contribution to the log likelihood can be written as

$$
\ln L_{i}=d_{i} \ln \left[h\left(t_{i}\right)\right]+\ln \left[S\left(t_{i}\right)\right]
$$

where $d_{i}=1$ for an event( i.e. death here) and 0 otherwise. For relative survival the survival and hazard functions are defined in equation 1 and 2 . This leads to

$$
\ln L_{i}=d_{i} \ln \left[h^{*}\left(t_{i}\right)+\lambda\left(t_{i}\right)\right]+\ln \left[S^{*}\left(t_{i}\right)\right]+\ln \left[R\left(t_{i}\right)\right]
$$

As $S^{*}\left(t_{i}\right)$ is not dependent on any of the unknown model parameters it can be removed from the log likelihood, thus,

$$
\ln L_{i}=d_{i} \ln \left[h^{*}\left(t_{i}\right)+\lambda\left(t_{i}\right)\right]+\ln \left[R\left(t_{i}\right)\right] .
$$

when using split-time data the term $\ln \left[R\left(t_{i}\right)\right]$ is the one that is split, into one for each follow-up interval. The current methods fit models on the log excess hazard scale i.e.

$$
h(t)=h^{*}(t)+\exp (\beta z)
$$

where $\beta$ is a vector of parameters to be estimated for covariates $z$. If we consider the hazard (2) then integrating both sides gives the cumulative hazard $H(t)$

$$
H(t)=H^{*}(t)+\Lambda(t)
$$

where $H(t)$ is the cumulative overall hazard $H^{*}(t)$ is the cumulative expected hazard and $\Lambda(t)$ is the cumulative excess hazard. We are interested in modelling on the log cumulative excess hazard scale for relative survival analysis:

$$
\ln (-\ln R(t ; z))=\ln (\Lambda(t))=\ln \left(\Lambda_{0}(t)\right)+\beta z
$$

where $\Lambda_{0}(t)$ is the baseline cumulative excess hazard function, $R(t ; z)$ is the relative survival function. Note that proportional cumulative excess hazards implies proportional 
excess hazards. Following Royston and Parmar we write $x=\ln (t)$ and the log cumulative excess hazard as:

$$
\ln (\Lambda(t))=s(x ; \gamma)+\beta z
$$

where $s(x ; \gamma)$ can be any smoothing function such as the various regression spline functions or fractional polynomials. For a proportional excess hazards model, let $\eta=s(x ; \gamma)+\beta z$. Then the associated relative survival and excess hazard functions are given by

$$
\begin{aligned}
R(t) & =\exp [-\exp (\eta)], \\
\text { and } \quad \lambda(t) & =\frac{1}{t} \frac{d s(x ; \gamma)}{d x} \exp (\eta)
\end{aligned}
$$

This implies that the overall survival is

$$
S(t)=S^{*}(t) \exp [-\exp (\eta)]
$$

with associated hazard

$$
h(t)=h^{*}(t)+\frac{1}{t} \frac{d s(x ; \gamma)}{d x} \exp (\eta)
$$

Substituting (10) and (11) into (5) gives the log-likelihood contribution for each subject,

$$
\ln L_{i}=d_{i} \ln \left[h^{*}\left(t_{i}\right)+\frac{1}{t} \frac{d s\left(x_{i} ; \gamma\right)}{d x_{i}} \exp \left(\eta_{i}\right)\right]-\exp \left(\eta_{i}\right)
$$

Time by covariate interactions are easy to include by modelling $\gamma_{i}$ separately for each covariate level, which removes the assumption of proportional excess hazards.

The model thus far has been generalisable as any smoothing function could be used for $s(x ; \gamma)$, but we follow the flexible parametric model for censored survival data that was proposed by Royston and Parmar[1] which incorporates the use of natural/restricted cubic 
splines in models on the cumulative hazard or cumulative odds scale (here we are just concerned with modelling on the cumulative excess hazard scale). Following Royston and Parmar the splines are defined by

$$
s(x ; \gamma)=\gamma_{0}+\gamma_{1} x+\gamma_{2} v_{1}(x)+\ldots+\gamma_{m+1} v_{m}(x)
$$

where the $j^{\text {th }}$ basis function is defined for $j=1, \ldots, m$ as:

$$
v_{j}(x)=\left(x-k_{j}\right)_{+}^{3}-\lambda_{j}\left(x-k_{\min }\right)_{+}^{3}-\left(1-\lambda_{j}\right)\left(x-k_{\max }\right)_{+}^{3}
$$

where $u_{+}=u$ if $u>0$ and $u_{+}=0$ if $u \leq 0, k_{\min }$ is the position of the first knot, $k_{\max }$ of the last knot and $\lambda_{j}=\frac{k_{\max }-k_{j}}{k_{\max }-k_{\min }}$, where the derivatives are obtained from (15) and (16) as

$$
\frac{d s(x ; \gamma)}{d x}=\gamma_{1}+\sum_{j=2}^{m} \gamma_{j}\left[3\left(x-k_{j}\right)_{+}^{2}-3 \lambda_{j}\left(x-k_{\min }\right)_{+}^{2}-3\left(1-\lambda_{j}\right)\left(x-k_{\max }\right)_{+}^{2}\right]
$$

Modelling is performed on the cumulative excess hazard scale as the survival and hazard functions can then both be estimated analytically and numerical integration is not required, which speeds up computation time. It is more likely to retain stability; for example, when modelling on this scale using log time and a Weibull distribution the relationship will be linear and as such departures from the Weibull will be under more stable conditions. This model does not use time-split data and expands upon a current method for 'standard' survival. The models are fitted in a Stata program available from the authors on request. The likelihood functions for the models were maximised using the Newton Raphson technique with the first and second derivatives estimated numerically as implemented using the ' $m l$ ' command in Stata[24]. 
The piecewise approach used in this paper use split-time data at the individual level, i.e. with many rows per person with the split times placed at: $0.5,1,2,3,4$, and 6 years. Piecewise models are fitted using the Dickman[18] approach using Poisson GLMs.

\section{DATA}

The dataset used for the analyses was set-up in 1987 at the Leicester Royal Infirmary in the UK. All admissions to the coronary care unit in Leicestershire, UK, are recorded and followed up to death. The data used in this paper are taken from 1993-2006 for consistency as there was a change in the database management as many more covariates were recorded from this time.

A total of 5859 observations include 2383 deaths (40.7\%) over the thirteen year period. Of these the cohort used for the analyses consists of 4748 (1760 deaths) observations and follow-up was censored after six years. Acute MI is described using characteristics of the electrocardiogram (ECG) and the results of blood tests. Only patients with ECG evidence of ST elevation, a criterion which did not change over our study period, are included. Moreover, ST elevation MI is associated with higher mortality. Thus, patients included are those experiencing their first known acute MI and demonstrating ST elevation on the ECG. Of the 4748 included in the analysis cohort there are 3231 men (68.1\%); 3965 (83.6\%) of the cohort self-reported as White and 763 Asian (16.1\%). The mean age of the cohort is 66.7 with men being 8 years younger than women on average, (64.1 :Men, 72.2 
:Women). 1759 (37.1\%) of this cohort died within the six year follow-up used in the investigation.

Heart disease has a very high increased mortality rate in the first 30 days, with perhaps $40-50 \%$ of all deaths occurring in this time period, there being 843 (47.9\%) for this cohort. The models need to capture this important feature of the data adequately. After admission for MI the risk of almost immediate death is high, yielding many deaths at day zero; 252 (4.7\%) in this cohort. Deaths on day zero was recorded as day 0.5.

Follow-up for analysis began at hospitalisation and not after 30 days survival as is the case with other heart disease mortality analyses[12]. The expected mortality was calculated using rates from the United Kingdom Government Actuary's Department[25] matched on age, sex and period of hospitalisation to the England and Wales population. We illustrate the methodology by estimating differences in relative survival/excess mortality by age group. We define our age groups as $<65$, $65-75$ and 75 years or older at entry.

There are two fitting principles used in this paper, one that uses split-time data at the individual level used in the piecewise approach and another that uses non-split time data which has one row of data for each individual with their survival time kept as continuous used in the flexible parametric approach.. 


\section{RESULTS}

\subsection{Piecewise Models}

A piecewise model was fitted using individual level split-time data with six time intervals on the time scale split annually for the 6 year follow-up, except for the first year which was split into two 6 month intervals and years 5 and 6 were combined. Using a proportional excess hazards $(\mathrm{PEH})$ assumption gave estimates of the excess hazard ratios (with 95\% confidence intervals) as $2.71(2.25,3.27)$ and $6.77(5.71,8.03)$ for $65-75$ and 75 years or older respectively using $<65$ age group as the comparative baseline. Both age groups have a significantly increased risk of death over the baseline group with the oldest age group being the worst. Extending the model to non-proportional excess hazards (non$\mathrm{PEH}$ ) by fitting an interaction between time interval and each covariate gave estimates for the excess hazard rate ratios as shown in table 1. This highlights a large increased uncertainty for the parameter estimates as follow-up time increases. There is a higher excess hazard ratio initially for both age groups. An excess hazard rate ratio up to 6 months of $7.30(6.06,8.79)$ for patients aged 75 years or older indicates that compared with a patient 65 years or younger they have an excess mortality rate that is over seven times higher during the first 6 months after MI. However, a likelihood ratio test between the PEH and non-PEH model showed a non-significant difference $\left(\chi_{10}^{2}=7.12, \mathrm{p}=0.714\right)$.

\subsection{Proportional excess hazard flexible parametric models}


The flexible parametric models using spline functions for the baseline cumulative excess hazard were fitted to the non-split heart disease data. The estimated relative survival curve with five internal knots split by age group adopting a PEH assumption are shown in figure 1. Vertical dashed lines on the figures indicate the position of the internal knots. Table 2 shows the Akaike information criterion (AIC) values for PEH models with different degrees of freedom along with estimates of the excess hazard rate ratios. Following Royston and Parmar the knot locations are based on percentiles of survival time for those that experience an event. All five models gave similar estimates for both excess hazard ratios. The PEH model with six degrees of freedom was determined as the optimal model using the AIC. Therefore the model was fitted using five internal knots placed at the 17, 33, 50, 67 and $83 \%$ percentiles of time of all deaths as adopted by Royston and Parmar[1]. The first two knots are fitted to day one and day four with knot three at day 44 and the last two knots are at day 439 and day 1074. The relative survival for the under sixty-five year olds is better than the 65-75 years group and much better than the over seventy-five years group. There is a large decrease in relative survival during the first six months and after this point the curves are still decreasing but at a slower rate. It is evident that the curves do not reach a plateau, suggesting that these patients do not achieve the same mortality rate as the general population to which they are compared. Figure 2 (a) shows the excess hazard rate compared to the standard piecewise approach described in section 4.1. The estimates of the model are in broad agreement. However the piecewise interval in the first 6 months does not capture the sharp reduction in the hazard rate adequately. The excess hazard shows the high excess mortality rate experienced by the three age groups, with the over 75 group having the highest excess mortality. Table 2 shows the estimate of the excess hazard ratio for the 6 
degrees of freedom model which is greater than one for both age-groups. The estimate of the excess hazard rate ratio for patients aged 65-75 years old is 2.58 which is similar to the piecewise model estimate (2.72). For patients aged 75 years or older the excess hazard rate ratio is estimated as 5.73 which is lower than the piecewise estimate of 6.80 .

4.3 Non-proportional excess hazard flexible parametric models

Using the same model selection criteria and using age groups as a covariate, a non-PEH model was fitted to the non-split data, which was obtained by fitting an interaction between time and a fixed covariate thus allowing the spline terms to vary by age group. The model with 6 degrees of freedom was again found to be the best of the non-PEH models using the AIC, as shown in table 2. Using the AIC values to compare the PEH and non-PEH models, for all but the 2-df model a PEH model would be selected. The excess hazard rate appears to give an adequate fit when compared to the standard piecewise non-PEH piecewise approach as shown in figure 2(b). By including six degrees of freedom for the spline terms and allowing age to vary over time, the estimated excess hazard rate for the youngest age group appears to stabilise earlier than in the PEH model in figure 2 (a). This is reflected by the fact that in figure 3 (a) and (b) the excess hazard rate ratios appear to increase and decrease sharply with a peak around the six month period. The estimates from the piecewise model assuming non-PEH have been overlaid on the figure and are in broad agreement with the estimates from the piecewise model.

Figure 4 provides an illustration of the various excess hazard rate ratios for models with three to six degrees of freedom. For the 65-75 age group (grey-lines) the 4, 5 and 6-df 
models show similar shapes with a large increase in risk for the first six months and then decreasing to an excess hazard rate ratio of approximately 1.5. The 3-df model does not capture any of the changes in excess hazard. Estimates for the over 75 group (black-lines) show a similar shape for all four models to begin with but the 4-df model decreases more than the other three models. However, it should be acknowledged that the time dependent effects are non-significant and there may be a degree of over-fitting here. 


\section{DISCUSSION}

Relative survival has advantages over the standard methods when dealing with population based censored survival data as the cause of death is not required. We have investigated the application of restricted cubic splines[1] on the cumulative excess hazard scale to allow modelling of time continuously and have compared these with piecewise models proposed by Dickman[18]. Age was categorised into three groups for both the piecewise and the proposed model, although there are advantages to also treating age as a continuous variable, we chose to categorise it for simplicity.

Although piecewise models are most popular in practice they are not clinically realistic which has led to interest in models that treat the timescale as continuous. To fit smooth functions of time fractional polynomials[19] and splines[20-22] have previously been used to model relative survival. These models are fitted on the log excess hazard scale, which requires either numerical integration or approximating the likelihood by splitting the data finely both of which can be computationally intensive. The model we use is fitted on the log cumulative excess hazard scale. The advantages of the extended Royston and Parmar model proposed here are that there is no splitting of the timescale, avoiding the need to arbitrarily choose split points, and the survival and hazard functions are estimated analytically and hence computationally quicker. As an illustration of computational time when using RSURV in R[26] which uses a PEH B-spline approach described by Giorgi et al.[21] using age groups and default values on our data converged in 148.58 seconds whereas our approach implemented in Stata using the same amount of knots (2 internal) also using a PEH assumption converged in 6.67 seconds and 5 internal 
knots with a non-PEH assumption converged in 27.86 seconds on a Pentium $4 \mathrm{CPU}$ 3.4Ghz PC. A key advantage is the ability to model the baseline hazard[27, 28]. A flexible parametric approach may provide an appropriate estimate for the baseline hazard whose behaviour may be of medical interest.

Using the AIC, a model with 5 internal knots was selected. However this investigation has shown various degrees of freedom in the spline models have produced very similar covariate effects using a PEH model. For these data there was no evidence to support rejection of the $\mathrm{PEH}$ model, either from the AIC or the likelihood ratio test for the piecewise models. The estimated coefficients were very similar amongst the various PEH models but different from the piecewise model estimates for the oldest age group of 6.60; all of the spline models estimated the excess hazard ratio in the range of 5.73 to 5.92 . If the piecewise model had more splits in the first 6-months then they become more similar, for example splitting the first 6 months into 5 intervals produced an estimate of 5.74. This shows that when the piecewise models are allowed to be more flexible the results become very similar to the spline results. However this requires more parameters to be estimated. A sensitivity analysis using the splines approach is always recommended especially in complex models. For PEH models the number and location of knots is unlikely to be crucial for estimating the excess hazard ratios, unless you miss the change in the first 6 month period i.e. miss the extremes.

There is an interest in relative survival models with time dependent effects. Flexible parametric models can be extended by allowing the covariate(s) to vary within each spline term by fitting an interaction. However in our dataset there is little evidence of 
non-PEH. If we were still interested in allowing the effect of age to vary over time then model selection becomes less intuitive as shown in figure 4. All of these models are modelling a similar effect but the 3-df and 4-df models do not appear to model the excess hazard rate ratios as accurately as required. Knot frequency and location is likely to be more important than in PEH models as illustrated in figure 4. However these differences should not be over interpreted for this particular case as the AIC favour PEH models. During the investigation of non-PEH models shown in figure 3 a sharp increase and decrease around the first 6 months were observed for the excess hazard rate ratios. This effect is likely due to youngest age group stabilising earlier and creating a larger difference earlier on around the 6 month period. Further sensitivity analyses were performed on non-PEH models varying the number and location of the knots. The method was found to be robust to small changes in the choice of knot location, for example fitting at 10, 30, 50 and $70 \%$ rather than the 20, 40,60 and $80 \%$ percentiles. This shows that the impact of knots depends on the structural form of the data and as such the type of data should be considered when fitting splines along with sensitivity analyses.

Relative survival in heart disease is in early development and there are limited examples in the literature of the use of relative survival methods in heart disease, although Stare et al.[29] has developed relative survival models using a heart disease dataset. Analyses conditional on survival for a month post MI[12], or conditional on discharge from hospital[29] do not have the problem of the very high excess hazard rate experienced in this time period. This is a key problem; if piecewise models were to be used then there is a requirement to split the timescale finely within the first few weeks to capture the shape. There are benefits of treating time as continuous, as the model proposed here does, but 
the high early mortality rate must be adequately modelled. It is important when modelling in observational studies of heart disease to investigate the effect of covariates in order to obtain adjusted estimates and investigate effect modifiers. It should also be noted that this approach is also suitable for other therapeutic areas including cancer. Here risk factors other than age associated with heart disease, such as sex and economic status have not been modelled, but these could be included in extended models.

The models could be extended in a number of ways. The models originally proposed by Royston and Parmar allowed the modelling of proportional hazards and proportional odds. Models for relative survival shown here have been fitted to the cumulative excess hazards scale, but could be extended to the odds scale. An automated selection of knot location may prove a useful extension but may result in clinically implausible fits[5]. The model could also be extended to other smoothing functions such as fractional polynomials. Assessment of the model fit is currently performed by overlaying the

piecewise estimates at the individual level over the spline models as shown in figure 2 . This along with the AIC is currently the main method of assessing the fit of the model and the criterion needs further attention.

\section{ACKNOLEDGEMENTS}

We thank Patrick Royston for his comments and involvement with the extension to the Royston and Parmar model and Paul Dickman for his contributions to discussions surrounding the methodology. We also thank the four referees for their useful comments 
and suggestions. Chris Nelson was funded by a British Heart Foundation PhD studentship (FS/05/080/19415). 


\section{REFERENCES}

1. Royston P, Parmar MKB. Flexible parametric proportional-hazards and proportional-odds models for censored survival data, with application to prognostic modelling and estimation of treatment effects. Statistics in Medicine 2002; 21(15):2175-2197.

2. Pillay D, Bhaskaran K, Jurriaans S, Prins M, Masquelier B, Dabis F, Gifford R, Nielsen C, Pedersen C, Balotta C, Rezza G, Ortiz M, Mendoza Cd, Ku“cherer C, Poggensee G, Gill J, Porter K. CASCADE Virology Collaboration: The impact of transmitted drug resistance on the natural history of HIV infection and response to first-line therapy. AIDS 2006; 20(1):21-28.

3. Griffin JT, Fraser C, Gras L, de Wolf F, Ghani AC. The Effect on Treatment Comparisons of Different Measurement Frequencies in Human Immunodeficiency Virus Observational Databases. American Journal of Epidemiology 2006; 163(7):676-683.

4. Royston P, Parmar MKB, Qian W. Novel designs for multi-arm clinical trials with survival outcomes with an application in ovarian cancer. Statistics in Medicine 2003; 22(14):2239-2256.

5. Royston P. st0001. Flexible parametric alternatives to the Cox model, and more. Stata Journal 2001; 1(1):1-28.

6. Ederer F, Axtell LM, Cutler SJ. The relative survival rate: a statistical methodology. National Cancer Institute Monographs 1961; 6:101-121.

7. Lauer MS, Blackstone EH, Young JB, Topol EJ. Cause of death in clinical research: Time for a reassessment? J Am Coll Cardiol 1999; 34(3):618-620.

8. Mant J, Wilson S, Parry J, Bridge P, Wilson R, Murdoch W, Quirke T, Davies M, Gammage M, Harrison R, Warfield A. Clinicians didn't reliably distinguish between different causes of cardiac death using case histories. Journal of Clinical Epidemiology 2006; 59(8):862-867.

9. Coleman M, Babb P, Damiecki P, Grosclaude P, Honjo S, Jones J, Knerer G, Pitard A, Quinn M, Sloggett A, Stavola BD. Cancer survival trends in England and Wales, 1971-1995: Deprivation and NHS region. Office for National Statistics, London. 1999.

10. Kvidal P, Bergstrom PR, Horte L-G, Stahle E. Observed and relative survival after aortic valve replacement. Journal of the American College of Cardiology 2000; 35(3):747-756.

11. Norman PE, Semmens JB, Lawrence-Brown MMD. Long-term relative survival following surgery for abdominal aortic aneurysm: a review. Cardiovascular Surgery 2001; 9(3):219-224.

12. Bronnum-Hansen H, Jorgensen T, Davidsen M, Madsen M, Osler M, Gerdes LU, Schroll M. Survival and cause of death after myocardial infarction: the Danish MONICA study. Journal of Clinical Epidemiology 2001; 54(12):1244-50.

13. Patel PJ, Keating RJ, Gersh BJ, Hodge DO, Hammill SC, Shen WK. Outcome of patients with newly diagnosed atrial fibrillation at the Mayo Clinic and residing in that area. 2004:1379-82.

14. Hardie K, Hankey GJ, Jamrozik K, Broadhurst RJ, Anderson C. Ten-year survival after first-ever stroke in the perth community stroke study. 2003:1842-6. 
15. Hankey GJ, Jamrozik K, Broadhurst RJ, Forbes S, Burvill PW, Anderson CS, Stewart-Wynne EG. Five-year survival after first-ever stroke and related prognostic factors in the Perth Community Stroke Study. 2000:2080-6.

16. Hakulinen T, Tenkanen L. Regression Analysis of Relative Survival Rates. Applied Statistics 1987; 36(3):309-317.

17. Esteve J, Benhamou E, Croasdale M, Raymond L. Relative survival and the estimation of net survival: elements for further discussion. Statistics in Medicine 1990; 9(5):529-38.

18. Dickman PW, Sloggett A, Hills M, Hakulinen T. Regression models for relative survival. Statistics in Medicine 2004; 23(1):51-64.

19. Lambert PC, Smith LK, Jones DR, Botha JL. Additive and multiplicative covariate regression models for relative survival incorporating fractional polynomials for time-dependent effects. Statistics in Medicine 2005; 24(24):38713885.

20. Remontet L, Bossard N, Belot A, Estève J, the French network of cancer registries FRANCIM. An overall strategy based on regression models to estimate relative survival and model the effects of prognostic factors in cancer survival studies. Statistics in Medicine 2007; 26(10):2214-2228.

21. Giorgi R, Abrahamowicz M, Quantin C, Bolard P, Esteve J, Gouvernet J, Faivre $\mathrm{J}$. A relative survival regression model using B-spline functions to model nonproportional hazards. Statistics in Medicine 2003; 22(17):2767-2784.

22. Bolard P, Quantin C, Abrahamowicz M, Esteve J, Giorgi R, Chadha-Boreham H, Binquet C, Faivre J. Assessing time-by-covariate interactions in relative survival models using restrictive cubic spline functions. Journal of Cancer Epidemiology \& Prevention 2002; 7(3):113-122.

23. Royston P, Altman DG, Sauerbrei W. Dichotomizing continuous predictors in multiple regression: a bad idea. Statistics in Medicine 2005; 25(1):127-141.

24. Gould W, Pitblado J, Sribney W. Maximum likelihood estimation with Stata: second edition. Stata Press 2003.

25. GAD. Interim life tables of mortality rates for England and Wales. http://www.gad.gov.uk/Life_Tables/Interim_life tables.htm; (accessed 30/10/2006).

26. Giorgi R, Payan J, Gouvernet J. RSURV: A function to perform relative survival analysis with S-PLUS or R. Computer Methods and Programs in Biomedicine 2005; 78(2):175-178.

27. Carstensen B. Who needs the Cox model anyway? http://staff.pubhealth.ku.dk/ bxc/frmain.html; (accessed 30/10/2006).

28. Hjort N. On inference in parametric survival data models. International Statistical Review 1992; 60:355 -387.

29. Stare J, Henderson R, Pohar M. An individual measure of relative survival. Applied Statistics 2005; 54(1):115-116. 


\section{TABLES}

\begin{tabular}{|c|c|c|c|c|c|c|}
\hline \multirow{2}{*}{$\begin{array}{l}\text { End of } \\
\text { interval } \\
6 \text { months }\end{array}$} & \multicolumn{3}{|c|}{$\begin{array}{c}\text { 65-75 years old } \\
\text { Excess hazard ratio }(\mathrm{Cl})\end{array}$} & \multicolumn{3}{|c|}{$\begin{array}{c}75 \text { years or older } \\
\text { Excess hazard ratio }(\mathrm{Cl})\end{array}$} \\
\hline & 2.85 & $(2.32$ & $3.50)$ & 7.30 & $(6.06$ & $8.79)$ \\
\hline Year 1 & 2.11 & $(\odot .78$ & $5.75)$ & 3.68 & (1.37 & $9.91)$ \\
\hline Year 2 & 2.18 & (1.05 & $4.49)$ & 3.93 & (1.89 & $8.14)$ \\
\hline Year 3 & $3.0 \odot$ & $(\odot .90$ & $9.98)$ & 5.98 & (1.81 & $19.80)$ \\
\hline Year 4 & 1.99 & $(\odot .61$ & $6.51)$ & 2.49 & $(\odot .51$ & 12.11) \\
\hline Year 5 \& 6 & 1.73 & $(0.46$ & $6.48)$ & 6.30 & $(2.17$ & $18.26)$ \\
\hline
\end{tabular}

Table 1 - Estimates of the excess hazard rate ratio with 95\% confidence intervals for the individual level piecewise non-proportional excess hazard model using split-time data.

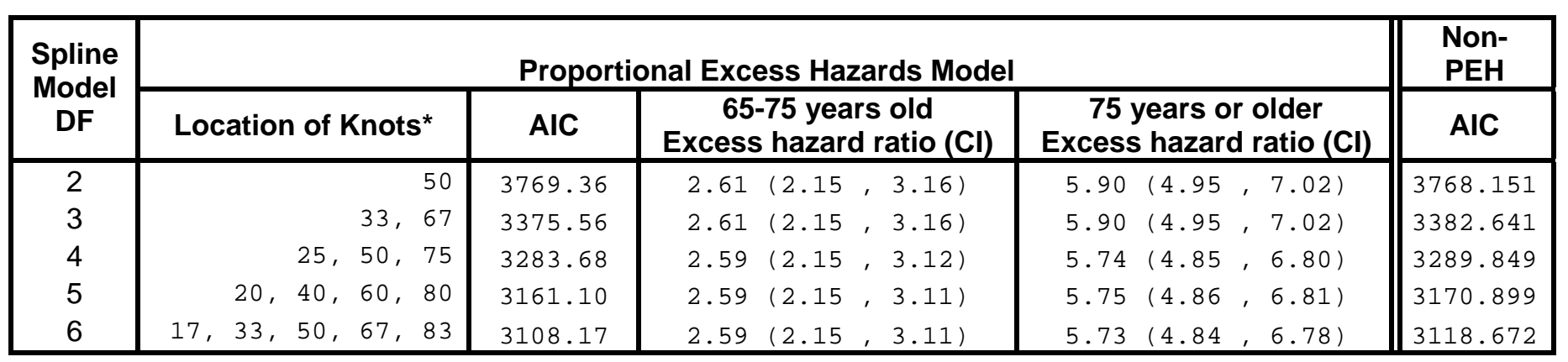

Table 2 - AIC and estimates of the excess hazard rate ratio with 95\% confidence intervals for spline models with various degrees of freedom using non-split data.

* Percentiles of all death times, as proposed by Royston and Parmar 


\section{FIGURES}

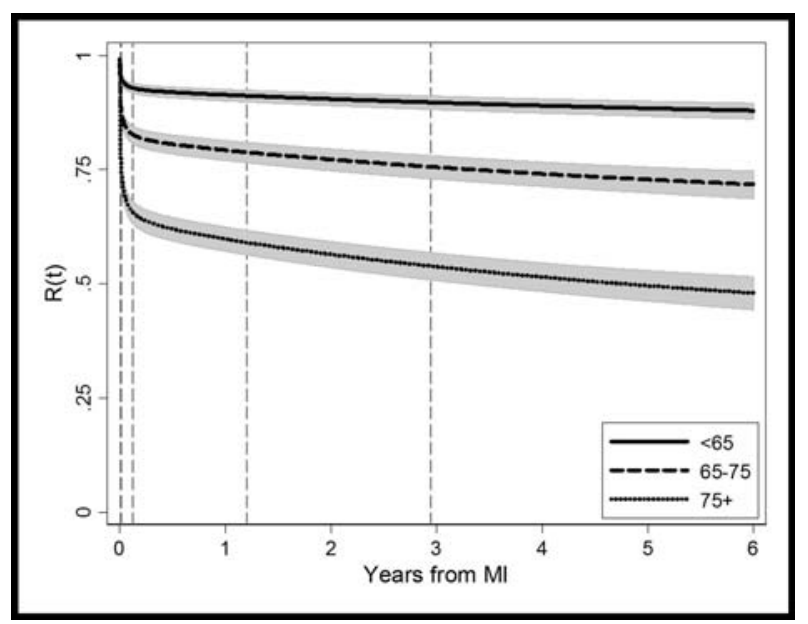

Figure 1 - Flexible parametric models using six degrees of freedom and a PEH assumption: the relative survival curve with 95\% confidence interval. Internal knots as shown by vertical dashed lines. It is not possible to distinguish the first 2 knot lines.

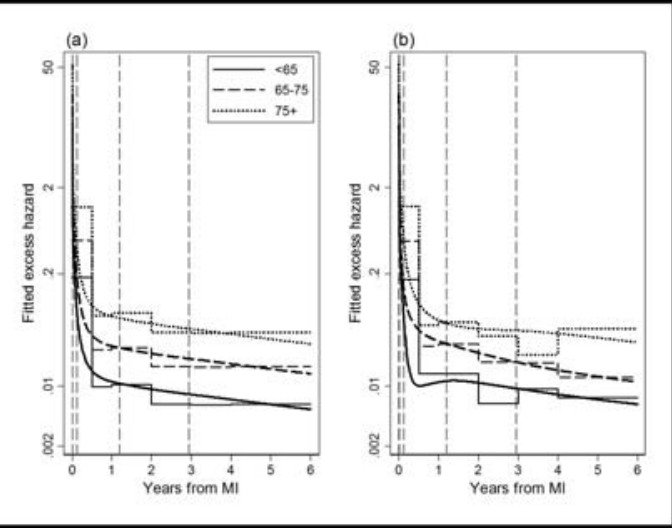

Figure 2 - Comparison of the fitted excess hazard rates for the selected spline model using non-split data with the piecewise individual level split-time data for (a) PEH and (b) non-PEH models. Internal knots as shown by vertical dashed lines. It is not possible to distinguish the first 2 knot lines. 


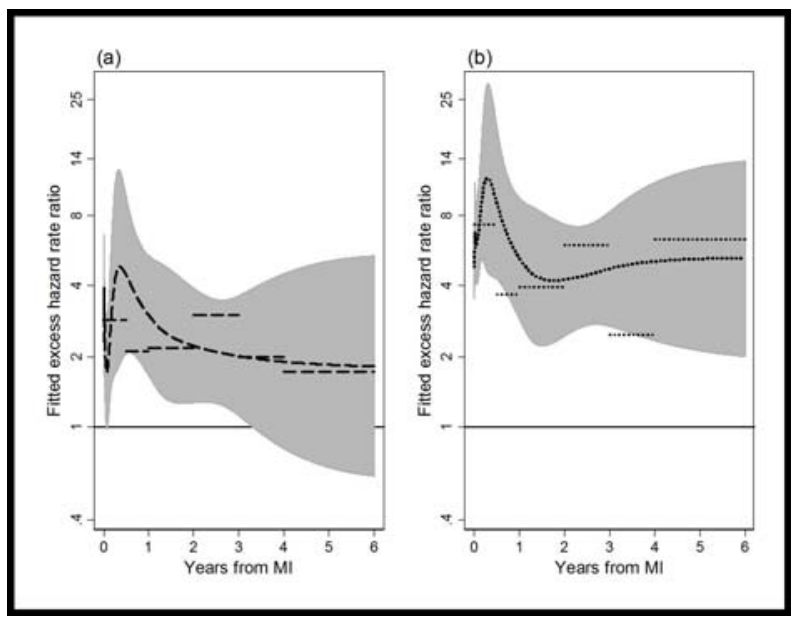

Figure 3 - The fitted excess hazard rate ratio with 95\% confidence interval alongside the piecewise estimates where - is an excess hazard rate ratio=1 for (a) aged 65-75 years and (b) aged 75+.

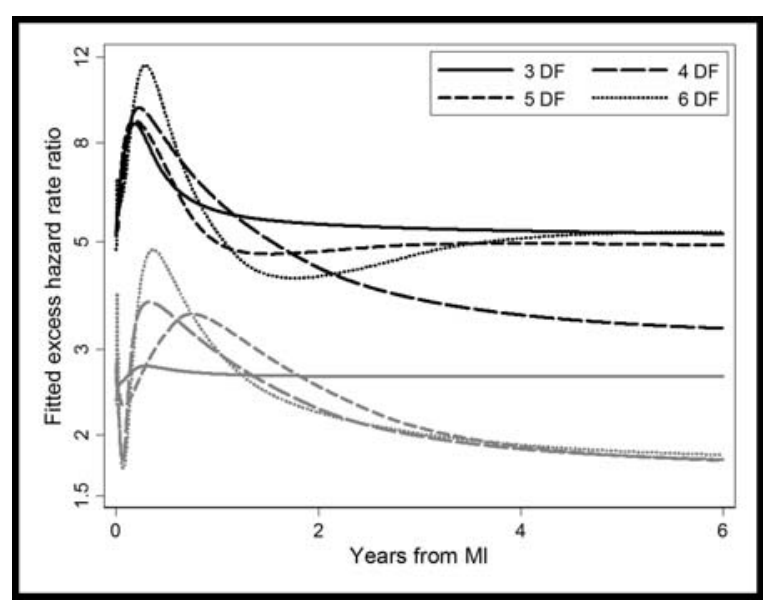

Figure 4 - Varying the degrees of freedom in the spline model and the effect on the fitted excess hazard rate ratio for patients aged 65-75 (grey) and patients aged 75 or older (black), both in comparison with patients aged 65 years or younger. 